\title{
Asymetric Telecollaboration in Virtual Reality
}

\author{
Thibault Porssut* \\ Ecole Polytechnique Fédéral de Lausanne, \\ Immersive Interaction Group, Switzerland
}

\author{
Jean-Rémy Chardonnet ${ }^{\dagger}$ \\ Le2i, Arts et Métiers, CNRS, Univ. \\ Bourgogne Franche-Comté, HeSam, \\ Institut Image, France
}

\begin{abstract}
We present a first study where we combine two asymetric virtual reality systems for telecollaboration purposes: a CAVE system and a head-mounted display (HMD), using a server-client type architecture. Experiments on a puzzle game in limited time, alone and in collaboration, show that combining asymetric systems reduces cognitive load. Moreover, the participants reported preferring working in collaboration and showed to be more efficient in collaboration. These results provide insights in combining several low cost HMDs with a unique expensive CAVE.
\end{abstract}

Keywords: Telecollaboration, CAVE, HMD.

Index Terms: H.5.1 [Information Interfaces and Presentation]: Multimedia Information Systems-Artificial, augmented, and virtual realities; H.5.3 [Information Interfaces and Presentation]: Group and Organization Interfaces-Computer-supported cooperative work

\section{INTRODUCTION}

The introduction of low cost virtual reality (VR) solutions, such as HTC Vive, allows considering new working methods, among which telecollaboration, for applications such as digital mockup reviews. We present a telecollaboration mode between two VR systems, aiming at improving users performance with reduced cognitive load.

Telecollaboration in VR has been explored in past research. One of the main constraints lies in the data transmission speed [7], which is less an issue now with for example optical fibers. Most of past work use CAVE systems for telecollaboration (e.g., [3]). The pros and cons of a two-person collaboration in a CAVE or with two HMDs for collaborative visualization of abstract data has been explored [1], showing comparable experiences in both systems, however, here, we do not look at comparing two systems, but at combining two different systems together, on a concrete task. Heldal et al. evaluated collaboration for solving a puzzle [5] but limited to evaluate the performance of two persons together, without knowing the performance of a person alone. Mortensen et al. realized a study on asymetric telecollaboration for a task requiring two persons, and showed a correlation between copresence and performance [6].

We present here a first study of telecollaboration between two persons using different VR systems: a CAVE system and a low cost HMD. The study aims at evaluating the interest of combining two different (asymetric) systems to improve users performance with reduced cognitive load. We hypothesized that combining asymetric systems in a collaborative way improves indeed users performance.

\section{Telecollaboration Architecture}

To achieve distant collaboration, we chose to define a server-client type architecture: the CAVE, a high cost, non transportable system,

\footnotetext{
*e-mail: thibault.porssut@epfl.ch

†e-mail: jean-remy.chardonnet@ensam.eu
}

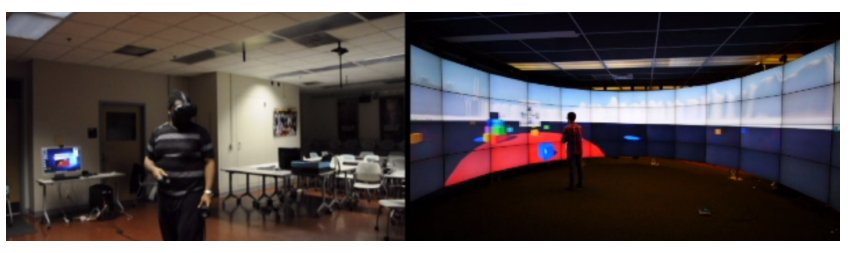

Figure 1: Collaborative puzzle construction session between a Vive user (left) and a CAVE2 user (right).

will be defined as the server whereas the HMD, a low cost, wearable device, thus easily duplicable, as a client.

\subsection{Hardware}

The CAVE used here is a CAVE2 ${ }^{\mathrm{TM}}$ [2], equipped with 72 LCD screens with a 37 megapixels total resolution and passive stereoscopy. We installed a microphone on the floor to let the user interact with a remote collaborator. On the contrary of more classic CAVEs, the CAVE2 does not have any projected floor, therefore full immersion is not possible.

The HMD used here is a HTC Vive, a low cost HMD, with a Lighthouse tracking system allowing to move naturally in a room. The user has two tracked controllers, one in each hand. A microphone is already integrated in the HMD.

\subsection{Software}

All the developments were made under Unity. Device networking was done using UNET, the Unity network add-on, while communication between the two remote users is achieved using Skype.

The application is a Puzzle Game, where the goal is to build a figure with virtual colored cubes placed in a virtual environment randomly (see Figure 1). The figure has to be built in a specific place (a red circle) in a limited time (10 min). Instructions are written on a virtual board. An application has been built for both systems.

In the CAVE2 version, the user holds a wand modeled by a hand in the game. He can navigate by walking within the physical space of the CAVE2, by teleportation or by using the wand's joystick. The virtual hand allows cube manipulation: when a cube is touched, it changes color. In the Vive version, users use both hands for manipulation and navigation can be achieved by walking or by teleportation (which is a good alternative to reduce cybersickness).

The differences between the CAVE2 and the Vive versions come from a pure industrial constraint. The CAVE2 is indeed delivered with only one wand with joystick-based displacements by default. Therefore, we wanted to respect basic technical constraints in order to simulate real configurations CAVE2 users usually face.

\section{Assessment Protocol}

18 people (10 men and 8 women, aged between 18 and 30) participated in the experiment ( 9 sessions, one per pair of participants).

A device, either the CAVE2 or the Vive, is assigned randomly to a participant and is kept by the participant throughout the experiment which is divided into three tasks: task 1 to get familiar with the device, task 2 where the participant is alone to complete 


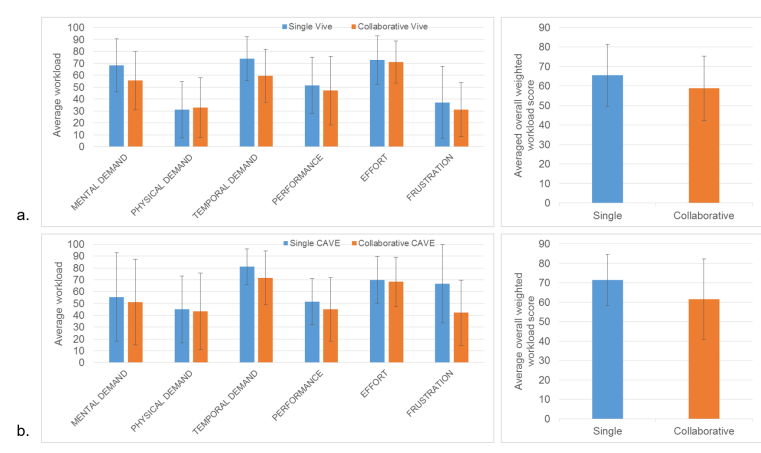

Figure 2: TLX for each task with: a. Vive, b. CAVE2.

the puzzle, and task 3 , where he completes the puzzle in remote collaboration with another participant. Both participants can speak to each other and see each other through avatar representation in the virtual scene. The figure to complete in each task is different to avoid learning effects.

We use both objective and subjective measures to evaluate the effect of collaboration. As subjective measures, we provide several questionnaires: one to know whether the participants have prior experience with VR and video games, potential handicaps and diseases that could affect their performance, and another as a feedback questionnaire on the experiment. We also use the NASATLX (Task Load Index) [4] to evaluate the different cognitive loads (mental, physical, temporal loads, effort, frustration and performance). As objective measures, we measure the completion rate: knowing the total number of cubes to place, we count the number of cubes a participant was able to place at the end of each session.

\section{Data Analysis}

\subsection{User Experience Questionnaire}

From the user experience questionnaire, half of the participants play video games regularly. Task 1 was designed to reduce the gap between participants (gamers and non-gamers).

\subsection{NASA-TLX}

We measured a TLX per task. The results in both situations (single - task 2 - and collaborative - task 3) are depicted in Figures 2.a and 2.b for participants with the Vive and the CAVE2 respectively.

The Friedman test shows that the effort demand either with the Vive or the CAVE2 is similar for both tasks $\left(\chi_{\text {Vive }}^{2}=0.50\right.$, $\left.\chi_{\text {CAVE2 }}^{2}=1.00, p>.05\right)$. Collaboration shows to have a significant effect on temporal $\left(\chi^{2}=5.44, p<.05\right)$ and mental demands $\left(\chi^{2}=4.50, p<.05\right)$ with the Vive. We suppose that the high level of frustration with the CAVE2 in task $2(M=66.7)$ comes from the absence of projected floor, complicating task accomplishment.

Hence, collaborative work allows reducing cognitive loads, thus reducing overall cognitive workload, significantly with the Vive $\left(\chi^{2}=4.50, p<.05\right)$. The participants felt more confident in collaboration than alone. Note that with the CAVE2, the workload did not reduce significantly, we suppose again that the CAVE2 configuration was not ideal and complicated the tasks.

\subsection{Completion Rate}

Figure 3 shows the completion rates for each task with each device. After conducting a one-way within-subject ANOVA, there was a significant effect of the task type (single or collaborative) on the performance : $F(2,24)=10.99, p=.0004$. Post-hoc comparisons showed that collaboration significantly improves performance, whatever the device (Vive: $t(8)=6.05, p=.0001$; CAVE2:

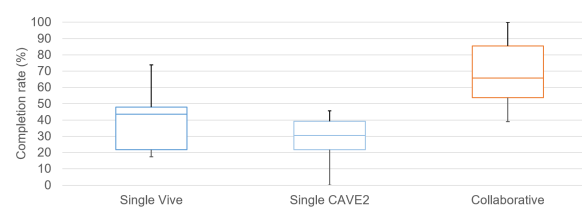

Figure 3: Completion rates for both tasks.

$t(8)=4.85, p=.0006)$. Note that from TLX measures, performance is perceived not to change significantly. Also we remarked that gamers did not perform better than non-gamers.

The difference in completion rate between tasks 2 and 3 is more important (but not in a significant way) for CAVE2 participants $(M=40.3)$ than for Vive participants $(M=28.0)$. It is possible that again one reason comes from the absence of projected floor in the CAVE2, forcing participants to move frequently and loose time.

\subsection{Feedback Questionnaire}

No major problem has been reported by the participants (Vive and CAVE2). Most of them (89\%) felt no or very little cybersickness.

We observed that overall, collaborative work is preferred to single work $(78 \%)$. Surprisingly, many CAVE2 participants $(28 \%)$ preferred working alone rather than in collaboration, on the contrary of Vive participants (all reported preferring working in collaboration). This observation can explain higher TLX indices for CAVE2 participants in both tasks.

\section{Conclusion}

We presented a first collaboration study using two asymetric systems. Collaboration allows reducing cognitive load. Completion rates show that users performance is enhanced, though from the TLX users reported a level of difficulty similar in both tasks.

We are willing to explore in a near future asymetric telecollaboration using different technologies for applications such as concurrent engineering, considering presence and embodiment.

\section{ACKNOWLEDGMENTS}

The authors wish to thank Mechdyne and the EVL at University of Illinois at Chicago for their help in using the CAVE2.

\section{REFERENCES}

[1] M. Cordeil, T. Dwyer, K. Klein, B. Laha, K. Marriott, and B. H. Thomas. Immersive collaborative analysis of network connectivity: Cave-style or head-mounted display? IEEE Trans. on Visualization and Computer Graphics, PP(99):1-10, 2016.

[2] A. Febretti, A. Nishimoto, T. Thigpen, J. Talandis, L. Long, J. D. Pirtle, T. Peterka, A. Verlo, M. Brown, D. Plepys, D. Sandin, L. Renambot, A. Johnson, and J. Leigh. Cave2: a hybrid reality environment for immersive simulation and information analysis. In SPIE, volume 8649 , pages 864903-864903-12, 2013.

[3] C. Fleury, T. Duval, V. Gouranton, and A. Steed. Evaluation of remote collaborative manipulation for scientific data analysis. In 18th ACM Symp. on Virtual Reality Software and Technology, pages 129136, 2012.

[4] S. G. Hart and L. E. Staveland. Development of nasa-tlx (task load index): Results of empirical and theoretical research. Advances in Psychology, 52:139-183, 1988.

[5] I. Heldal, A. Steed, and R. Schroeder. Evaluating collaboration in distributed virtual environments for a puzzle-solving task. In 11th International Conference on HCI, 2005.

[6] J. Mortensen, V. Vinayagamoorthy, M. Slater, A. Steed, B. Lok, and M. C. Whitton. Collaboration in tele-immersive environments. In EGVE '02, pages 93-101, 2002.

[7] V. S. Pantelidis. The rave, cave, and collaborative virtual environments. Technical report, East Carolina University, 2000. 